\title{
Competing magnetic anisotropies in atomic-scale junctions
}

\author{
Alexander Thiess, ${ }^{1}$ Yuriy Mokrousov, ${ }^{1}, *$ and Stefan Heinze ${ }^{2}$ \\ ${ }^{1}$ Institut für Festkörperforschung, Institute for Advanced Simulation, Forschungszentrum Jülich, D-52425 Jülich, Germany \\ ${ }^{2}$ Institute of Theoretical Physics and Astrophysics, Christian-Albrechts-University of Kiel, D-24098 Kiel, Germany
}

(Received 27 November 2009; published 22 February 2010)

\begin{abstract}
Using first-principles calculations, we study the magnetism of $5 d$ transition-metal atomic junctions including structural relaxations and spin-orbit coupling. Upon stretching monoatomic chains of W, Ir, and Pt suspended between two leads, we find the development of strong magnetism and large values of the magnetocrystalline anisotropy energy (MAE) of up to $30 \mathrm{meV}$ per chain atom. We predict that switches of the easy magnetization axis of the nanocontacts upon elongation should be observable by ballistic anisotropic magnetoresistance measurements. Due to the different local symmetry, the contributions to the MAE of the central chain atoms and chain atoms in the vicinity of the leads can have opposite signs which reduces the total MAE. We demonstrate that this effect occurs independent of the chain length or geometry of the electrodes.
\end{abstract}

DOI: 10.1103/PhysRevB.81.054433

PACS number(s): 73.63.Nm, 72.25.-b, 75.30.Gw, 75.47.-m

\section{INTRODUCTION}

Fascinating insights into the formation of atomic chains consisting only of a few atoms suspended between two electrodes have been obtained by transmission electron microscopy and mechanically controllable break junction techniques. ${ }^{1-3}$ These experiments triggered the imagination of scientists to use such atomic-scale junctions as future electronic devices by exploiting their unique properties, e.g., ballistic electronic transport. ${ }^{1,4,5}$ Due to their reduced dimension, atomic chains have also been predicted to develop magnetic moments even for elements nonmagnetic in bulk such as Ir, Pt, or Pd. ${ }^{6,7}$ Recently, an indirect proof has been given that transition-metal (TM) chains in break junctions are in general magnetic. ${ }^{8}$ Mastering the intriguing magnetic properties of suspended chains would enable spintronic applications based on the unique possibility to probe, control, and switch the magnetic states by spin-polarized currents. ${ }^{9,10}$

The orientation of the magnetic moments is stabilized against thermal fluctuations by the magnetocrystalline anisotropy energy (MAE) arising from spin-orbit coupling (SOC). Theoretical studies of this key quantity performed for idealized systems such as free-standing infinite monowires (MWs) and small clusters suggest giant values for $4 d$ - and $5 d$-transition metals and switching of the easy axis upon stretching the wires. ${ }^{11-13}$ Even the size of the magnetic moment itself can crucially depend on the magnetization direction, an effect coined as colossal magnetic anisotropy. ${ }^{4}$ The ballistic conductance in such wires varies with the orientation of the magnetization direction with respect to the chain axis giving rise to ballistic anisotropic magnetoresistance. ${ }^{1,5}$ While the predicted effects probably occur in real atomicscale junctions, the theoretical studies have either focused on idealized systems or neglected varying interatomic distances in the chains. ${ }^{14-16}$ However, for small suspended chains variations in the interatomic distance due to stretching the electrodes are unavoidable and can provide a unique way to control the properties of the system, not available in higher dimensions. ${ }^{4,6,11,17}$

Here, we go beyond such theoretical studies by explicitly taking the contacts into account and by investigating the de- velopment of magnetism in atomic-scale junctions upon increasing the distance between the electrodes. We perform first-principles calculations for W, Ir, and Pt junctions including spin-orbit coupling and structural relaxations and focus on atomic chains of three atoms suspended between two bulklike bcc-(001) electrodes as shown in Fig. 1. These transition metals are common tip materials in scanning tunneling microscopy, and Ir and Pt have been shown to form long atomic chains in break junction experiments. ${ }^{2}$ In particular, we concentrate on the influence of varying tip-to-tip separation on the magnetic properties of the junctions. We find that relaxations of the chain atoms upon stretching are in accordance to the observed experimentally trends of chain forma-

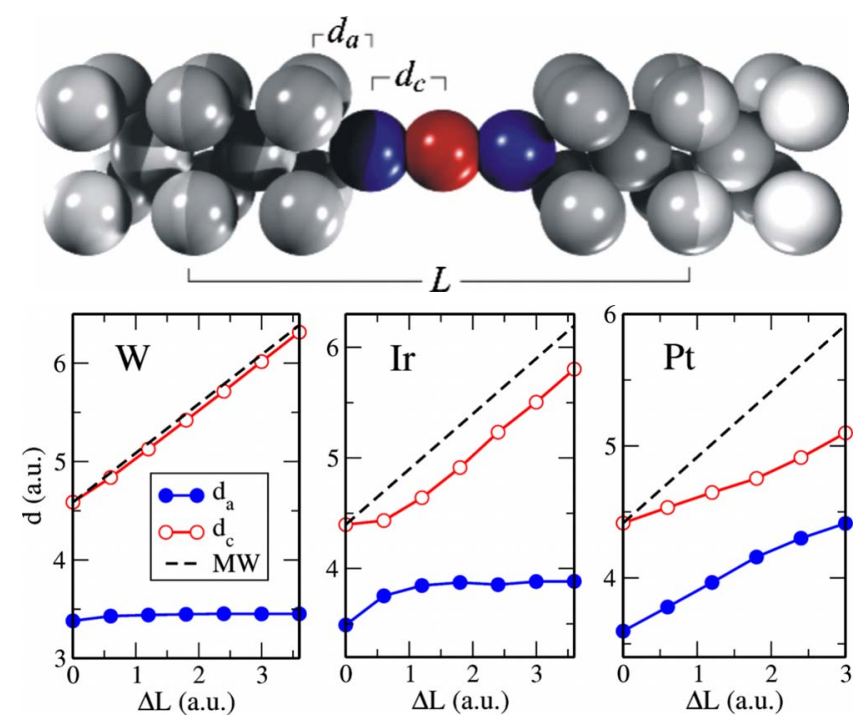

FIG. 1. (Color online) Upper panel: three-atoms chain suspended between two bcc-(001) contacts. $L$ denotes the length of the unit cell consisting of 17 atoms while $d_{a}$ and $d_{c}$ give the distance between the first plane of the contact to the apex atom and from the apex atom to the central atom of the trimer, respectively. Lower panel: relaxation of the $d_{c}$ and $d_{a}$ bonds in suspended trimers of $\mathrm{W}$, Ir, and Pt upon increasing $L$. For comparison, the bond length for ideal stretching with fixed apex atom positions is given (dashed line). 
tion for these elements. ${ }^{17}$ We also find that large spin moments develop in the suspended trimers upon stretching and on the central atom they reach similar values as in infinite monowires. ${ }^{6-8}$ Interestingly, the $\mathrm{W}$ trimer behaves as a single spin impurity as only the central atom develops a sizable magnetic moment.

By including SOC, we obtain the MAE of the suspended chains and observe large values of 10-30 meV per atom. Surprisingly, we find that the contributions to the MAE from central chain atoms and atoms in contact with the electrodes can have opposite signs and are of similar giant magnitude of a few tens meV per atom. The magnitude of this effect, unknown in bulk or on surfaces may lead to a strong tendency toward noncollinear magnetic order in atomic-scale junctions even when the exchange interactions are small or profoundly collinear. ${ }^{18}$ We prove the generality of this phenomenon by showing that it occurs for chains of different length and for different contact geometries. Further, the MAE may exhibit changes of sign upon stretching, i.e., the easy magnetization direction switches from along the chain axis to perpendicular to it. We demonstrate that switching may be observable experimentally by measuring the ballistic conductance of the junctions.

\section{METHOD AND STRUCTURE}

We have performed first-principles calculations within the generalized gradient approximation to the density-functional theory. We employed the full-potential linearized augmented plane-wave method for one-dimensional (1D) systems, ${ }^{19}$ as implemented in the FLEUR code. ${ }^{20}$ Basis functions were expanded up to $k_{\max }=4.0$ a.u. $^{-1}$ and we have used $8 k$ points in one half of the 1D Brillouin zone. Considering more $k$ points does not change the values of the spin moments and magnetic anisotropy energies. The contact structure has first been relaxed separately in all three dimensions and then kept fixed for all contact separations $L$ (Fig. 1). The apex atoms of the trimer have been relaxed along the chain direction $(z)$ upon varying $L$ while the central atom is fixed by symmetry (Fig. 1). Calculations have been performed for junctions stretched from the equilibrium up to $\Delta L=3$ a.u. For Pt and Ir trimers we considered the ferromagnetic ground state while $\mathrm{W}$ trimer always converges to the antiferromagnetic solution.

Spin-orbit coupling was included into the calculations in a nonperturbative way. ${ }^{21} \mathrm{We}$ define the magnetic anisotropy energy (MAE) as the total-energy difference between configurations with a magnetization along the chain axis $(z$ direction) and perpendicular to it ( $r$ direction). The MAE was calculated employing the force theorem, and for several electrode stretchings, $\Delta L$, we checked the force theorem results for the trimer's MAE against self-consistent calculations, finding good qualitative agreement. Taking into account the magnitude of the obtained MAE we can safely neglect the magnetic dipole-dipole contribution.

In order to check the applicability of our 1D geometry for the electrodes, which allows us to efficiently perform the demanding calculations with structural relaxations and including SOC, we have also considered a geometry with electrodes represented by surfaces, c.f. Fig. 2(a). Due to the im-
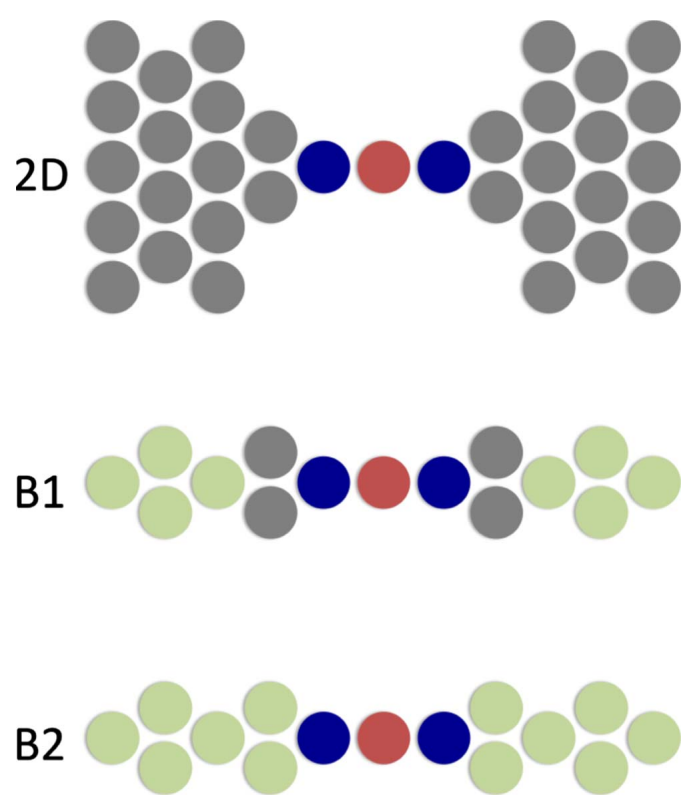

FIG. 2. (Color online) Surface contact 2D configuration, and B1 and $\mathrm{B} 2$ cases of the $\mathrm{Pt}$ trimer considered for the separation of $\mathrm{Pt}$ electrodes of $\Delta L=2.4$ a.u. and 1.8 a.u., respectively. In the $2 \mathrm{D}$ configuration the trimer is suspended between two electrodes consisting of a four Pt atom square base deposited onto three layers of fcc Pt(001). Green color (light filled circles) in B1 and B2 marks the Pt atoms of the contacts in which the spin moment was artificially quenched. In accordance to Fig. 1, red color marks the central atom and the blue color marks the apex atoms of suspended trimer. For more details see text.

mense computational cost, we have restricted our test to the most critical case of a Pt trimer and chose an elongation of $\Delta L=2.4$ a.u. For these calculations we used the film version of the FLEUR code. We performed a single supercell calculation in which the trimer was suspended between two electrodes consisting of a four Pt atom square base deposited onto three layers of fcc $\mathrm{Pt}(001)$. This configuration will be referred to as $2 \mathrm{D}$ in the following, Fig. 2. The ideal bulk values of $\mathrm{Pt}$ with an in-plane lattice constant of 5.23 a.u. were taken for the four contact atoms and the bulklike leads. The positions of the trimer's atoms were set to their relaxed values at $\Delta L=2.4$ a.u. obtained within the wire geometry of Fig. 1. The in-plane separation between the neighboring trimers was set to 15.7 a.u. We used a basis functions cutoff of $k_{\max }=3.7$ a.u. $^{-1}$ and $15 k$ points in the irreducible part of the two-dimensional Brillouin zone while $144 k$ points in the whole Brillouin zone were used in order to determine the MAE with the force theorem. Along the trimer's axis only the $\Gamma$ point was used.

\section{RELAXATIONS AND MAGNETISM}

Under the condition of reduced coordination of transitionmetal atoms in a monoatomic chain suspended between thicker leads, a situation realized, e.g., in a break junction experiment, the distance $d$ between the chain atoms becomes of utter importance. Upon pulling apart the leads during the chain elongation process ${ }^{3,17} d$ is constantly changing which 
has dramatic consequences for the magnetic properties of suspended chains, in particular, for heavy $4 d$ and $5 d$ transition metals. ${ }^{4,6,7,11}$ By increasing the separation $\Delta L$ between the leads and accounting at the same time for the changes in the chain's interatomic distances-which was neglected in most of the previous $a b$ initio studies of magnetism in suspended chains-we aim at mimicking a real break junction experiment.

The relaxations of the trimers along the chain's symmetry $z$ axis are shown in Fig. 1 , where the bond lengths $d_{c}$ and $d_{a}$ are presented as a function of contact separation $L$. For W with an almost half-filled $5 d$ shell and large bulk cohesive energy almost the entire increase in contact separation enters the $d_{c}$ bond length while the $d_{a}$ bond between the apex atom and the contact is nearly unaffected. In contrast, for Ir and Pt, some of the stretching transfers into an increased bond length at the contact. The apex atom of the Ir trimer has a stronger bond to the contact than to the central atom, which is reflected in the linear regime of $d_{c}(L)$ for $\Delta L>1.5$ a.u. For Pt, the $d_{c}$ bond is significantly stronger resulting in a remarkable stretching of the $d_{a}$ bond even at large electrode separations, which in a realistic break junction will eventually result in chain elongation. ${ }^{17}$ Overall, our predicted trend is in accordance with experimental observations of increased probability for chain formation when going from $\mathrm{W}$ to $\mathrm{Pt}^{2,22}$

The magnetic properties of suspended $5 d$ TM atoms are very sensitive to the local environment. In most previous studies it was common to model the contacts by semi-infinite surface electrodes. ${ }^{14-16}$ This restriction on the geometry of the contacts is quite strong, in particular, for $5 d$ TM break junctions, as the surface geometry leads to suppressed spin moments of the atoms in suspended wire and contact atoms closest to it. ${ }^{14}$ However, it is well established that the shape of the contacts in a break junction is rather a thinning wirelike geometry with a reduced coordination number of the contact atoms adjacent to the chain which greatly enhances their tendency toward magnetism. ${ }^{23,24}$ In this respect our contact geometry is probably closer to the real situation, although it might overestimate the magnetism of the contacts. In order to investigate this point quantitatively, we have performed calculations in the setup shown in Fig. 2, which are discussed further below.

The calculated spin moments $\mu_{S}$ inside the atomic spheres of the suspended trimers upon increasing the distance between the contacts $\Delta L$ are shown in Fig. 3. For W, the central atom reveals a sizable magnetic moment of $1.7 \mu_{B}$ already at $\Delta L=0$ which further increases upon stretching, while the moments of all other atoms are negligible. In this respect the $\mathrm{W}$ trimer presents a unique system of a single spin impurity between nonmagnetic leads. At small stretching, the spin moments of the Ir trimer are rather small with significantly larger moments of the central atom. Upon further stretching the apex and central moments rise rapidly, reaching large values of $1.4 \mu_{B}$ and $2 \mu_{B}$, respectively. Both, the $\mu_{S}^{c}$ spin moments of the central W and Ir chain atoms follow the behavior in an infinite monowire (MW) very closely as seen in Figs. 3(a) and 3(b).

The Pt trimer shows a trend similar to Ir upon stretching, only with smaller moments - this is in contrast to an infinite Pt MW, where the spin moment is zero for a large interval of
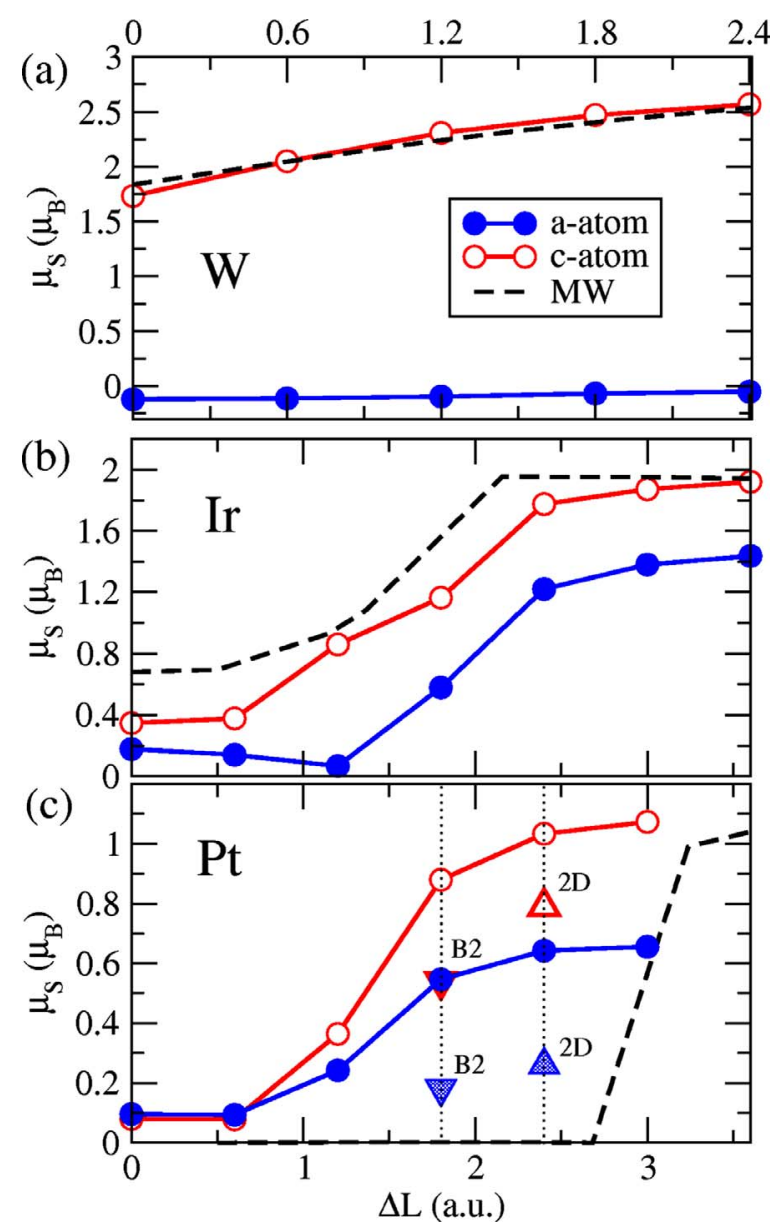

FIG. 3. (Color online) Local spin moments of the central $\left(\mu_{S}^{c}\right)$ and apex $\left(\mu_{S}^{a}\right)$ atoms (denoted as "c-atom" and "a-atom," respectively) of the suspended (a) W, (b) Ir, and (c) Pt trimers as a function of the stretching of the contacts (without SOC). For comparison the values for an infinite free monowire (MW) are given by dashed lines. The interatomic distance in the infinite MW corresponds to the distance $d_{c}$ for a given $\Delta L$. For Pt, up and down triangles stand for the values of the apex (filled) and central (open) spin moments in the B2 and 2D cases, respectively (cf. Fig. 2). For details see text.

interatomic distances without SOC. It is resurrected upon including SOC, but only when the magnetization points along the $z$ axis, an effect coined as colossal magnetic anisotropy. ${ }^{4}$ In contrast, for the suspended Pt trimer, the moments of the trimer atoms are nonzero already without SOC for a wide range of $d_{c}$. Upon including SOC the spin moments do not change dramatically and the moments are nonzero for both magnetization directions in the whole range of $\Delta L$, which manifests the subtle magnetism of Pt chains and its sensitivity to local environment.

In the critical case of $\mathrm{Pt}$, we analyze in more detail the influence of the contact geometry on the spin moments of the trimer. At the stretching of $\Delta L=1.8$ a.u. we performed a calculation in which we artificially quenched the spin moments in the contacts by applying a small magnetic field inside the muffin-tin spheres of the Pt atoms, a situation referred to as "B2" in the following (see Fig. 2). We observe that $\mu_{S}^{a}$ drops significantly from $0.55 \mu_{B}$ to $0.18 \mu_{B}$ while $\mu_{S}^{c}$ 
drops from $0.89 \mu_{B}$ to $0.54 \mu_{B}$ [Fig. 3(c)] —a value, very close to that reported in Ref. 14, where contacts were modeled by infinite bulk electrodes. However, quenching the spin moment in the deeper parts of the contacts only ("B1" case, Fig. 2) - the situation which is probably closer to that in real experiments-almost does not affect the values of $\mu_{S}^{c}$ and $\mu_{S}^{a}$.

Finally, we compare the results obtained within our 1D geometry of the electrodes with the situation of surfacelike electrodes, as shown in Fig. 2(a). For a separation of $\Delta L$ $=2.4$ a.u. $\mu_{S}^{c}$ is reduced in the $2 \mathrm{D}$ geometry by only $25 \%$ from $1.03 \mu_{B}$ in the wire geometry to $0.79 \mu_{B}$, while the moment of the apex atom is affected stronger, dropping from $0.64 \mu_{B}$ to $0.26 \mu_{B}$ [Fig. 3(c)]. In a more realistic situation the coordination of the contact atoms next to the apex atoms is more reduced and the moments of the trimer's atoms will increase approaching the values obtained in our wire geometry. However, we have to conclude that magnetism in suspended short Pt chains with their small moments is extremely sensitive to slightest changes in the contact geometry. Therefore, observing this magnetism experimentally will be a hard task, even considering the cluster's large magnetic anisotropy energies.

\section{MAGNETIC ANISOTROPY ENERGIES}

Recent theoretical studies, ${ }^{17,25}$ which are in accordance with experiments, ${ }^{3,26}$ clearly state that the interatomic distance $d_{c}$ in suspended chains of $5 d$ elements consisting of several atoms can reach large values of 5.0-5.5 a.u. upon stretching, which corresponds to $\Delta L \approx 2-3$ a.u., c.f. Fig. 1 . Therefore, in a real Pt, Ir, or W break junction experiment the central atom will reveal strong fingerprints of spin polarization at sufficiently small temperatures. In order to study the thermal stability of magnetism in the suspended trimers, we now concentrate on their magnetic anisotropy energies.

In a transport break junction experiment, the data is obtained by averaging over thousands of measurements, which differ from each other by the thinning history of the wire and the contact geometry. Therefore, in order to analyze this data with respect to the magnetism of the suspended chain, it is necessary to disentangle the contributions of intrinsic trimer's magnetic anisotropy energy $\Delta E^{\text {tri }}$ from that originating from the contacts. In our calculations, we do this by switching SOC off in the contact's atomic spheres. Moreover, we individually switch SOC on and off in the apex and central atoms, to determine their separate contributions, $\Delta E^{a}$ and $\Delta E^{c}$, respectively, to the total MAE of the trimer, $\Delta E^{\text {tri }}$. For $\mathrm{W}$ with essentially one magnetic atom in the trimer we define $\Delta E^{\text {tri }}$ as $\Delta E^{c}+2 \Delta E^{a}$ while for Ir and Pt we define the total MAE of the trimer per atom as $\Delta E^{\text {tri }}=\left(\Delta E^{c}+2 \Delta E^{a}\right) / 3 .{ }^{27}$

The absolute value of trimer's MAE, shown in Fig. 4 with a black solid line for $\mathrm{W}$, Ir, and $\mathrm{Pt}$, reach large absolute values of $10-30 \mathrm{meV}$ per atom at most electrode separations. These values, which are one to two orders of magnitude larger than those in most of transition-metal nanostructures, give us confidence that the magnetism of suspended trimers can be tackled experimentally. For all three elements the calculated values of anisotropy energies $\Delta E^{\text {tri }}$ are on the order of magnitude of those predicted for infinite monowires
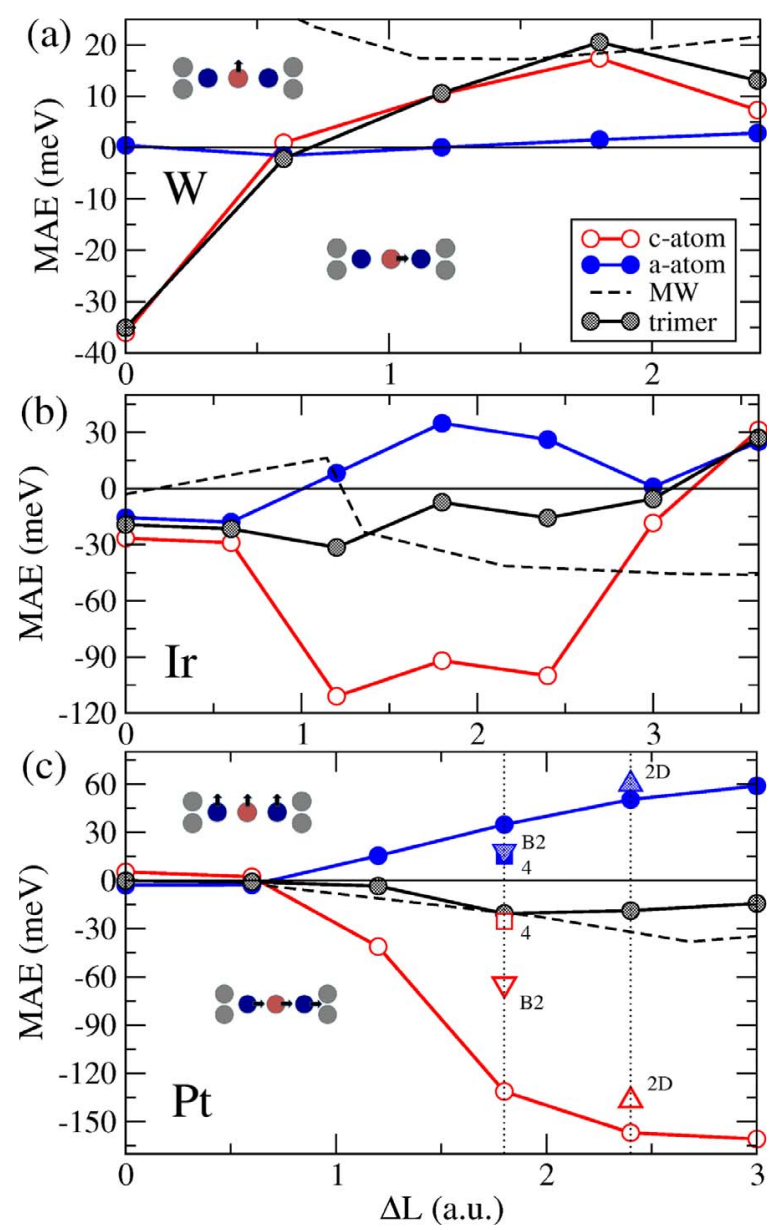

FIG. 4. (Color online) Magnetic anisotropy energies of suspended trimers of (a) W, (b) Ir, and (c) Pt as a function of the stretching of the contacts $\Delta L$. The total trimer's anisotropy $\Delta E^{\text {tri }}$ (black half-filled circles) is decomposed into the contribution from the central $\Delta E^{c}$ (red open circles) and apex atoms $\Delta E^{a}$ (blue filled circles) (for definitions of the quantities see text). Black dashed line stands for the MAE of the infinite MWs (per atom). For Pt, squares denote $\Delta E^{a}$ (filled) and $\Delta E^{c}$ (open) tetramer values while up and down triangles stand for the corresponding values in the $\mathrm{B} 2$ and $2 \mathrm{D}$ cases, respectively. For details see text.

(dashed line in Fig. 4 and Refs. 4 and 11). Interestingly, the trimer's MAE displays a nontrivial dependence on $\Delta L$ quite different from that in ideal chains for Ir and $\mathrm{W}$ while for $\mathrm{Pt}$ both anisotropy energies are close in their trend.

For W, Fig. 4(a), the only contribution to $\Delta E^{\text {tri }}$ stems from the strongly spin-polarized central atom. Starting at about 35 $\mathrm{meV}$ and a magnetization along the chain, there is a switch to a perpendicular direction upon stretching by $\Delta L=0.6$ a.u. Upon further increasing the contact separation, the perpendicular magnetization is stabilized by a sizable MAE of 10-20 meV. A switch of the magnetization direction can be also observed for Ir at significant stretching of around $\Delta L$ $=3.0$ a.u., Fig. 4(b). In general, for Ir, the behavior of $\Delta E^{\text {tri }}$ upon stretching is quite smooth, and the $z$ direction of the magnetization is favored by considerable $15-30 \mathrm{meV}$ over a wide interval of contact separations.

Detecting traces of magnetism in Pt break junctions at small stretching will present a significant challenge as can be 
seen from Fig. 4(c). In the regime of $\Delta L<1.3$ a.u. the value of $\Delta E^{\text {tri }}$ is on the order of a few meV, posing the question of whether the sensitive magnetization will survive temperature fluctuations. Moreover, in this regime, as pointed out above, the spin moments of the apex and central atoms depend strongly on contact geometry and details of the thinning process. This will influence the contribution of these atoms to $\Delta E^{\text {tri }}$, causing frequent measurement-to-measurement oscillations in its sign and magnitude. Beyond a distance of $\Delta L$ $=1.5$ a.u. the well-developed magnetization of the trimer is pointing rigidly along the chain axis with a MAE of about 15 $\mathrm{meV}$ - a value somewhat smaller than that of an infinite MW of corresponding interatomic distance, c.f. Fig. 4(c).

As a general trend, we observe in Fig. 4 competing contributions to the MAE from central and apex atoms for Ir and Pt atomic junctions. At most separations a positive value from the apex atom, $\Delta E^{a}>0$, favors a perpendicular magnetization direction, while negative contribution from the central atom, $\Delta E^{c}<0$, forces the magnetization along the trimer's axis. Due to this competition the trimer's MAE behaves qualitatively differently from that in the infinite MW as a function of the interatomic distance for Ir. It significantly quenches the central atom's MAE so that the resulting total anisotropy is on average smaller than anticipated from the infinite MW both for Ir and Pt.

The origin of this competition is the different local symmetry of apex and central atoms and it exists also in longer chains or chains made of other elements. To demonstrate this point we additionally calculated the MAE of a suspended four-atom chain of $\mathrm{Pt}$ atoms with the contacts as in Fig. 1. The $\Delta E^{a}$ and $\Delta E^{c}$ anisotropy energies for the two apex and two central atoms, respectively, are displayed by squares in Fig. 4(c) (per atom) and reveal the same effect: $\Delta E^{a}$ and $\Delta E^{c}$ are close in their values but opposite in sign. Due to its large magnitude, this effect may even compete with exchange interactions in suspended small chains and lead to a noncollinear magnetic order. With increasing chain length the anisotropy energies of the atoms in the center of the chain will eventually approach that of an atom in the infinite monowire [this can be already seen in Fig. 4(c) for Pt when going from a trimer to a tetramer] and the whole cluster will behave as a superparamagnet. For smaller suspended clusters, however, which are much more probable to occur in an experiment, the effect of competition between central and apex atoms will be dramatic.

We demonstrate that the effect of the competition between the apex and central atoms for the MAE value is also stable with respect to the geometry of the contacts. For this purpose we calculate the MAE of the Pt trimer in the 2D configuration, as well as in the B1 and B2 cases, and present the calculated values in Fig. 4(c). As far as the 2D case is concerned, we observe that despite significant changes in the spin moments, c.f. Fig. 3(c), the values of $\Delta E^{a}$ and $\Delta E^{c}$ are very close to those calculated within the wire geometry, moreover, they are also opposite in sign. Their competition results in rather close values for the total $\Delta E^{\text {tri }}$ of 7 and 18 $\mathrm{meV}$ for the 2D and wirelike geometry of the contacts, respectively, both favoring the $z$ direction of the trimer's magnetization. Notably, both values are very close to that of 12 $\mathrm{meV}$ reported by Smogunov et al. in Ref. 14 for the Pt trimer

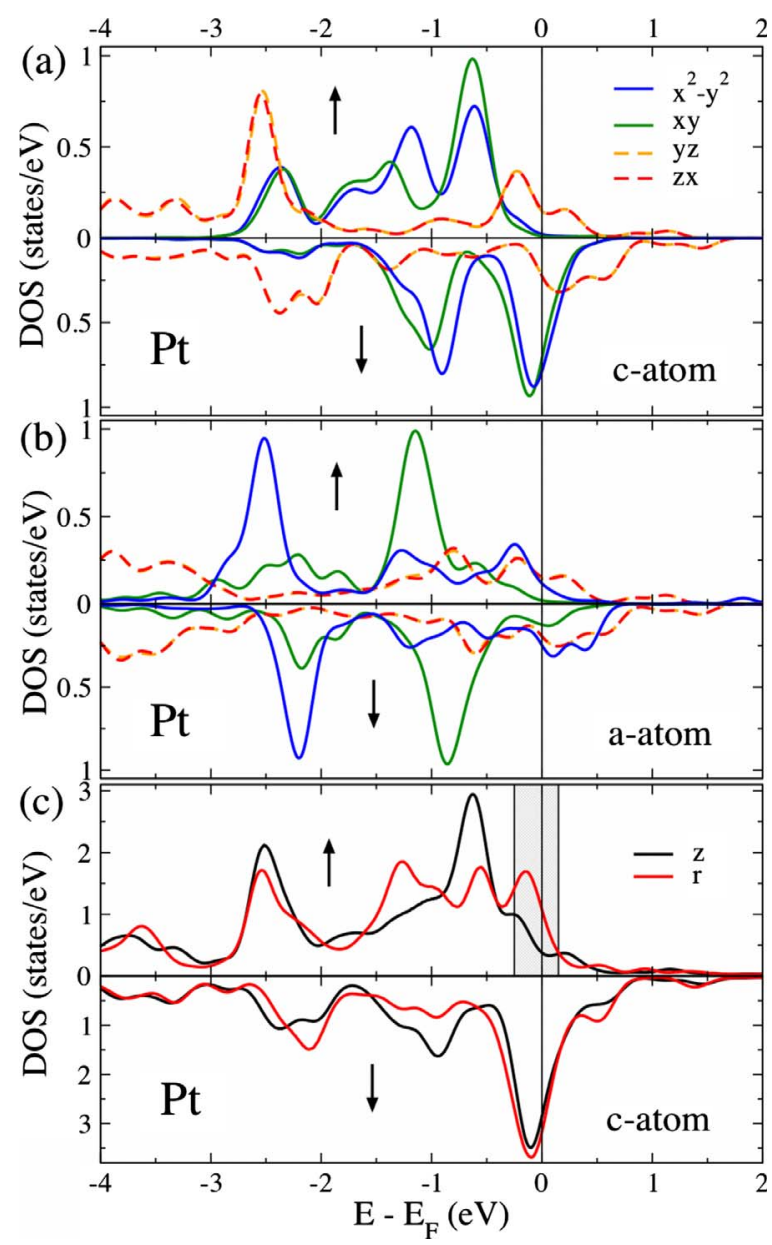

FIG. 5. (Color online) Spin-decomposed LDOS for a Pt trimer including SOC for a separation between the electrodes of $\Delta L$ $=1.8$ a.u. [(a) and (b)]: $\Delta_{3}\left(d_{x z}, d_{y z}\right)$ and $\Delta_{4}\left(d_{x^{2}-y^{2}}, d_{x y}\right) 3 d$ LDOS of the central (c-atom) and apex (a-atom) atoms for the magnetization along the $z$ axis. (c) LDOS of the central atom for two different magnetization directions $(z$ and $r$ ).

suspended between semi-infinite electrodes, although one has to keep in mind the difference between the geometries of all three cases.

In the B1 situation, in which we do not quench the spin moments of the contact atoms of the electrode, the MAE is almost not affected compared to the wire setup of Fig. 1. On the other hand, in the B2 case, in which we quench the spin moments inside the entire electrode, the spin moments of the trimer atoms are strongly reduced, c.f. Fig. 3(c), and so is the MAE: $\Delta E^{c}$ and $\Delta E^{a}$ drop to almost half their value. However, the latter two contributions to the total MAE $\Delta E^{\text {tri }}$ are still of opposite sign, proving the generality of the phenomenon of competing anisotropies for this type of atomic junctions. The latter effect is responsible for the value of the total MAE $\Delta E^{\text {tri }}$ of $9 \mathrm{meV}$, smaller than the value of $20 \mathrm{meV}$ obtained within the wire geometry.

To understand the effect, we analyze the local density of states (LDOS) and choose the Pt trimer as an example. In an infinite $\mathrm{MW}$, the position of the localized $d$ states with respect to the Fermi energy $\left(E_{F}\right)$ is responsible for the formation of the orbital moment and the direction of the 
magnetization. ${ }^{11,28,29}$ According to the symmetry these $d$ states can be subdivided into $\Delta_{3}\left(d_{x z}, d_{y z}\right)$ and $\Delta_{4}\left(d_{x^{2}-y^{2}}, d_{x y}\right)$ groups, not taking into account the $\Delta_{1} s d$ hybrid. ${ }^{11}$ In a Pt (Ir) infinite MW, the position of the $\mathrm{X}$ edge ( $\Gamma$ edge) of the $\Delta_{4}$ degenerate $d_{x y}$ and $d_{x^{2}-y^{2}}$ bands at $E_{F}$ is responsible for the easy magnetization direction along the chain. ${ }^{28,29}$ In a trimer at larger stretching, the $5 d$ LDOS of a central atom qualitatively follows that of an atom in an infinite monowire (not shown), which explains the same sign of the MAE (Fig. 4). A small splitting between the $d_{x^{2}-y^{2}}$ and $d_{x y}$ states due to the presence of the leads can be already seen for the central atom [Fig. 5(a)], however, for the apex atom the effect is dramatic and has crucial consequences for the apex MAE. The interaction with the contacts locally breaks the $C_{1 \infty}$ symmetry of an infinite chain which results in large splitting between the $d_{x y}$ and $d_{x^{2}-y^{2}}$ orbitals in the $\Delta_{4}$ band and their shift toward the lower energies [Fig. 5(b)]. On the other hand, the degeneracy between the $d_{x z}$ and $d_{y z}$ states $\left(\Delta_{3}\right.$ band) is locked by symmetry. This leads to the rearrangement of the states around $E_{F}$ and as a result the in-plane direction of the apex spin moment becomes favorable (Fig. 4).

In general, it should be possible to deduce the predicted switches in the magnetization direction of the suspended chains from transport measurements in these systems. As an example, in Fig. 5(c) we present the LDOS of the central atom in a Pt trimer at $\Delta L=1.8$ a.u. Upon changing the magnetization direction significant changes in the LDOS around the Fermi energy $E_{F}$ can be observed [shaded area in Fig. $5(\mathrm{c})$ ], which will inevitably result in large changes in the experimentally measured conductance. As far as the $5 d$ TMs are concerned, giant values of the ballistic anisotropic magnetoresistance should be achievable due to strong spin-orbit coupling in these metals, which is able to modify the electronic structure significantly in response to the changes in the magnetization direction in real space.

\section{CONCLUSIONS}

By performing $a b$ initio calculations of suspended trimers of W, Ir, and Pt including structural relaxations as a function of the separation between the leads, we demonstrate that the chain atoms develop significant spin moments upon stretching. We investigate the stability of these spin moments via evaluating the magnetic anisotropy energy of the trimers. Our calculations reveal large MAE of the whole trimers on the order of $10-30 \mathrm{meV}$ per atom. Interestingly, we observe that the total MAE presents a competition between large contributions from the apex and central atoms. We argue that this effect is general and can occur in suspended chains of different elements and different length, leading to nontrivial real-space textures of magnetic anisotropy energy which might even lead to complex magnetic ordering in atomicscale junctions.

Under the condition of large predicted values of MAE huge magnetic fields would be required to change the magnetization of the chains, therefore, new ways to achieve this goal have to be established. One of the possibilities lies in using the ability to control the magnetization direction in the junction by changing the distance between the leads. We show that such switches of the magnetization can happen and will result in strong features in the measured conductance. Distinguishing these features from the changes in conductance due to chain elongation or atomic rearrangements presents a considerable challenge.

\section{ACKNOWLEDGMENTS}

Financial support of the Stifterverband für die Deutsche Wissenschaft is gratefully acknowledged. We also thank Phivos Mavropoulos for discussions.
*Corresponding author. y.mokrousov@fz-juelich.de

${ }^{1}$ A. Sokolov, C. Zhang, E. Y. Tsymbal, and J. Redepenning, Nat. Nanotechnol. 2, 171 (2007).

${ }^{2}$ R. H. M. Smit, C. Untiedt, A. I. Yanson, and J. M. van Ruitenbeek, Phys. Rev. Lett. 87, 266102 (2001).

${ }^{3}$ T. Kizuka, Phys. Rev. B 77, 155401 (2008).

${ }^{4}$ A. Smogunov, A. Dal Corso, and E. Tosatti, Nat. Nanotechnol. 3, 22 (2008).

${ }^{5}$ J. Velev, R. F. Sabirianov, S. S. Jaswal, and E. Y. Tsymbal, Phys. Rev. Lett. 94, 127203 (2005).

${ }^{6}$ A. Delin and E. Tosatti, Phys. Rev. B 68, 144434 (2003).

${ }^{7}$ A. Delin, E. Tosatti, and R. Weht, Phys. Rev. Lett. 92, 057201 (2004).

${ }^{8}$ A. Thiess, Y. Mokrousov, S. Heinze, and S. Blügel, Phys. Rev. Lett. 103, 217201 (2009).

${ }^{9}$ P. Lucignano, R. Mazzarello, A. Smogunov, M. Fabrizio, and E. Tosatti, Nature Mater. 8, 563 (2009).

${ }^{10}$ M. R. Calvo, J. Fernández-Rossier, J. J. Palacios, D. Jacob, D. Natelson, and C. Untied, Nature (London) 458, 1150 (2009).

${ }^{11}$ Y. Mokrousov, G. Bihlmayer, S. Heinze, and S. Blügel, Phys.
Rev. Lett. 96, 147201 (2006).

${ }^{12}$ T. O. Strandberg, C. M. Canali, and A. H. MacDonald, Phys. Rev. B 77, 174416 (2008).

${ }^{13}$ L. Fernández-Seivane and J. Ferrer, Phys. Rev. Lett. 99, 183401 (2007).

${ }^{14}$ A. Smogunov, A. Dal Corso, and E. Tosatti, Phys. Rev. B 78, 014423 (2008); J. Fernández-Rossier, D. Jacob, C. Untied, and J. J. Palacios, Phys. Rev. B 72, 224418 (2005); D. Jacob, J. Fernández-Rossier, and J. J. Palacios, Phys. Rev. B 77, 165412 (2008).

${ }^{15}$ K. M. Smelova, D. I. Bazhanov, V. S. Stepanyuk, W. Hergert, A. M. Saletsky, and P. Bruno, Phys. Rev. B 77, 033408 (2008).

${ }^{16}$ M. Czerner, B. Y. Yavorsky, and I. Mertig, Phys. Rev. B 77, 104411 (2008)

${ }^{17}$ A. Thiess, Y. Mokrousov, S. Blügel, and S. Heinze, Nano Lett. 8, 2144 (2008).

${ }^{18}$ S. Mankovsky, S. Bornemann, J. Minár, S. Polesya, H. Ebert, J. Staunton, and A. Lichtenstein, Phys. Rev. B 80, 014422 (2009).

${ }^{19}$ Y. Mokrousov, G. Bihlmayer, and S. Blügel, Phys. Rev. B 72, 045402 (2005) 
${ }^{20} \mathrm{http}: / / w w w . f l a p w . d e$

${ }^{21}$ C. Li, A. J. Freeman, H. J. F. Jansen, and C. L. Fu, Phys. Rev. B 42, 5433 (1990).

${ }^{22}$ C. Untiedt, M. J. Caturla, M. R. Calvo, J. J. Palacios, R. C. Segers, and J. M. van Ruitenbeek, Phys. Rev. Lett. 98, 206801 (2007).

${ }^{23}$ L. G. C. Rego, A. R. Rocha, V. Rodrigues, and D. Ugarte, Phys. Rev. B 67, 045412 (2003).

${ }^{24}$ D. Cheng, W. Y. Kim, S. K. Min, T. Nautiyal, and K. S. Kim,
Phys. Rev. Lett. 96, 096104 (2006).

${ }^{25}$ E. Z. da Silva, A. J. R. da Silva, and A. Fazzio, Phys. Rev. Lett. 87, 256102 (2001).

${ }^{26}$ S. B. Legoas, D. S. Galvão, V. Rodrigues, and D. Ugarte, Phys. Rev. Lett. 88, 076105 (2002).

${ }^{27}$ In reality the value of the apex MAE is obtained by subtracting the MAE of the central atom from the MAE of the whole trimer. ${ }^{28}$ G. van der Laan, J. Phys.: Condens. Matter 10, 3239 (1998).

${ }^{29}$ A. Thiess, Y. Mokrousov, and S. Heinze (unpublished). 